\title{
A FAST APPROACH FOR COUPLED FLUID-THERMAL MODELING OF THE LUBRICATING INTERFACES OF AXIAL PISTON MACHINES
}

\author{
Swarnava Mukherjee*, Lizhi Shang, Andrea Vacca \\ Maha Fluid Power Research Center, Purdue University, USA \\ * Corresponding author: Tel.: +1 (765) 496-1283; E-mail address: mukher25@purdue.edu
}

\begin{abstract}
The temperature distribution of the lubricating interfaces is an important aspect of the functioning of positive displacement machines. It can determine the efficiency and the life time of the unit. In particular, it directly affects the fluid properties and the thermal induced deformations of the solid bodies. A simulation tool capable of predicting the fluid temperature in such gaps thus becomes very useful in the design process of these machines. The temperature distribution in a film comprises of many physical phenomena including convection and conduction along and across the film. Past numerical approaches solved this multi-directional conduction-convection problem using a threedimensional(3D) grid, making the tool computationally expensive and unsuitable for fast simulations. This paper proposes a hybrid fluid temperature solver, based on, a low computational cost twodimensional(2D) grid, to reduce the simulation time with reasonable accuracy. The piston/cylinder interface of an axial piston machine is selected as reference case to demonstrate the proposed approach. The hybrid approach was found to speedup the simulation times by $36 \%$.
\end{abstract}

Keywords: Heat transfer, Lubrication, Piston/cylinder interface, Energy equation

\section{INTRODUCTION}

Lubricating interfaces form a very critical area when designing axial piston machines. They have a direct impact on critical performance parameters like energy efficiency and load carrying ability. The evaluation of the lubricating features of these interfaces is rather complicated due to several physical phenomena that occur through them. The pressure in these gaps can reach very high values owing to the loading these gaps balance. Due to the micron level thickness of these gaps, a considerable amount of viscous dissipation occurs, leading to high temperatures. Both pressure and temperature in the lubricating film directly affect the gap dimension of these films, owing to pressure as well as thermal induced deformations of the bounding solid bodies. The design of these interfaces through simplified numerical procedures and trial and error-based methods often is very expensive due to several design iterations. Thus, it becomes very important to develop simulation tools capable of accurately predict the performance of these interfaces.
Among all the different physical phenomena occurring in these interfaces, the thermal behavior is one of the most important ones to consider. The temperature in the interfaces directly affects the viscosity, the density and the thermal induced deformations of the bounding solids. Predicting the temperature of the film poses a challenge in terms of the problem size. The energy transport equation needs to be solved in the film to predict the temperature distribution in the film[1]. This equation is a $3 \mathrm{D}$ equation. The three-dimensions pose a numerically large problem to solve, which affects the simulation time. With the desirable goal of bringing simulation tools in within the design processes, the simulation time is a very important factor. At the same time, since the calculation of the gap fluid pressure distribution is in a $2 \mathrm{D}$ domain, the fluid viscosity and density field that is calculated using the 3D temperature distribution are always averaged into a $2 \mathrm{D}$ field. This will be further explained in the following paragraphs.

The goal of this paper is to propose a method to solve the energy equation in two dimensions, drastically reducing the size of the problem and 
speed up the simulation time. The assumptions and simplifications are rationalized in the following sections.

Although the proposed approach is of general applicability to other lubricating interfaces, to demonstrate the proposed energy equation model, the piston/cylinder interface of an axial piston machine of swashplate type is chosen as reference. The piston/cylinder interface is one of the most important lubricating gaps in an axial piston machine owing to its tight clearances and its fundamental load carrying function. The first numerical simulation of pressure distribution in the piston/cylinder interface was conducted by Van der Kolk[2]. This was a simplified model considering a tilted journal bearing. The motion of the piston was firstly considered by Yamaguchi[3]. The first non-isothermal fluid model for the piston/cylinder interface was introduced by Ivantysynova[4] which addressed the non-constant fluid properties in the fluid film. Olems[5] first considered the piston micromotion from the force balance between external loads and the pressure in the interface. Wieczorek et al.[6] introduced the simulation tool CASPAR, a computer aided optimization tool capable of predicting the pressure and temperature behavior in all the lubricating interfaces of an axial piston machine. Huang et al.[7] later introduced the effect of elasto-hydrodynamic lubrication in the piston/cylinder interface utilizing an offline influence matrix approach to evaluate the pressure deformations. Pelosi et al.[8] introduced the first coupled fluid-structure-thermal interaction model for the piston/cylinder interface accounting for the pressure and thermal deformations. Pelosi et al.[9] further updated this model for accurate prediction of pressure distribution in the film. Shang et al.[10] introduced a method to predict the port flow temperatures to make the previous models completely independent from requiring measurement data. Shang et al.[11] later introduced a coupled heat transfer model for the accurate evaluation of the solid body temperatures. The temperature distribution in the lubricating film of a plain journal bearing was studied by McCallion et al.[12]. A comparison between numerical and experimental thermodynamic behavior of a journal bearing was conducted by Ferron et al.[13]. A numerical study of the film temperature and its effect on the performance of the piston/cylinder interface of an axial piston machine was performed by Song et al.[14].

An overview of the state of the art fluid structure thermal interaction model developed by Pelosi et al.[8] is shown in Figure 1. In this model, the 2D pressure and 3D temperature distributions are solved for, in the fluid domain using the finite volume approach. The pressure and gap heights are solved accounting for the fluid-structure interaction through pressure deformations, followed by solving the energy equation. The heat flux from the fluid film is averaged over one shaft revolution to solve for the solid body temperature distribution followed by solid body thermal deformation. These temperatures and deformations are then used as updated boundary conditions for solving the fluid pressure and temperature in the following revolution.

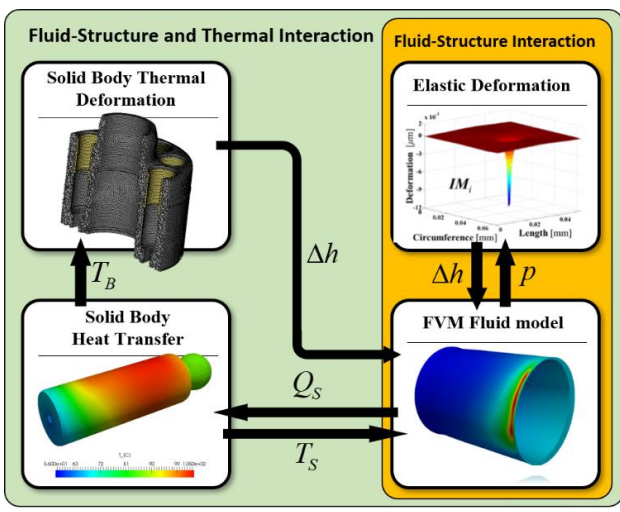

Figure 1 Fluid Structure Thermal interaction model

The current paper builds on the works of Wieczorek et al.[6] and Pelosi et al.[15] to introduce the mentioned 2D energy equation, for the simulation model to be more adoptable into iterative design and optimization processes.

\section{3D FLUID DOMAIN THERMAL MODEL}

The energy equation is provided in Eq.(1). This equation is solved once the Reynolds equations is solved. The velocity in the first term of Eq.(1) is derived using a combination of the pressure field and the velocity of the piston running surface 
contributing to both the Couette and the Poiseuille components.

$$
\rho c_{p} \vec{V} \cdot \vec{\nabla} \mathrm{T}-\vec{\nabla} \cdot(\lambda \vec{\nabla} T)=\mu \Phi_{D}
$$

In the previous approach[6], Eq.(1) was discretized using the finite volume approach on a grid similar to that shown in Figure 2. The fluid domain between the bore and piston is discretized in the circumferential, axial as well as the radial direction. The grid shown in Figure $\mathbf{2}$ is unwrapped circumferentially such that the circumference is along the $x$-axis, the axial direction is along the y-axis and the z-axis is aligned along the film thickness. Figure 3 shows one finite volume cell from the unwrapped fluid domain. $T_{E}, T_{W}, T_{N}, T_{S}, T_{T}$ and $T_{B}$ represent the temperatures of the east, west, north, top and bottom neighbor temperatures of a given cell temperature $T_{P}$. The respective walls of the cell are represented by e, $w, n, s, t$ and $b . \Delta x, \Delta y$ and $\Delta z$ represent the dimensions of the cell along the $\mathrm{x}, \mathrm{y}$ and $\mathrm{z}$ axis.

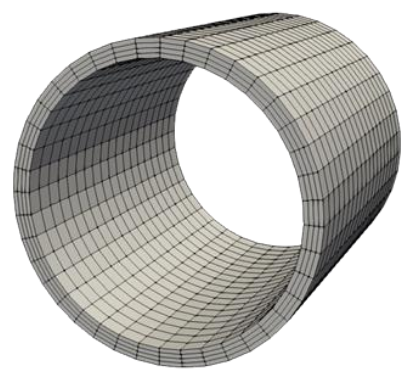

Figure 2 3D FVM grid

The discretized form of Eq.(1) for the cell shown in Figure 3 is shown in Eq.(2). The coefficients are shown in Eq.(3) to Eq.(10).

$$
\begin{gathered}
a_{E} T_{E}+a_{W} T_{W}+a_{N} T_{N}+a_{S} T_{S} \\
+a_{T} T_{T}+a_{B} T_{B} \\
+b=a_{P} T_{P} \\
a_{E}=D_{x} \cdot \max \left(0,\left(1-\frac{0.1\left|F_{x}\right|}{D_{x}}\right)^{5}\right) \\
+\max \left(0,-F_{x}\right)
\end{gathered}
$$

$$
\begin{aligned}
a_{W}=D_{x} \cdot \max & \left(0,\left(1-\frac{0.1\left|F_{x}\right|}{D_{x}}\right)^{5}\right) \\
+ & \max \left(0, F_{x}\right)
\end{aligned}
$$

$$
\begin{gathered}
a_{N}=D_{y} \cdot \max \left(0,\left(1-\frac{0.1\left|F_{y}\right|}{D_{y}}\right)^{5}\right) \\
+\max \left(0,-F_{y}\right)
\end{gathered}
$$

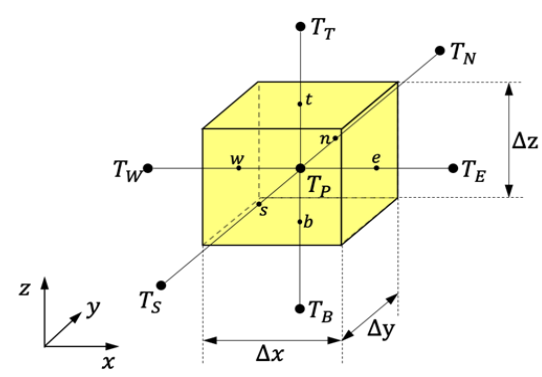

Figure 3 FVM cell from 3D grid

$$
\begin{gathered}
a_{S}=D_{y} \cdot \max \left(0,\left(1-\frac{0.1\left|F_{y}\right|}{D_{y}}\right)^{5}\right) \\
+\max \left(0, F_{y}\right) \\
a_{T}=D_{z} \\
a_{B}=D_{z} \\
b=\mu \Phi_{D} \Delta x \Delta \mathrm{y} \Delta \mathrm{z} \\
a_{P}=a_{E}+a_{W}+a_{N}+a_{S}+a_{T} \\
+a_{S}
\end{gathered}
$$

$D_{x}, D_{y}$ and $F_{x}, F_{y}$ represent the diffusive and convective coefficients along the $\mathrm{x}$ and $\mathrm{y}$ axis respectively. $D_{z}$ represents the diffusive coefficients along the $\mathrm{z}$-axis. The expressions for these are shown in Eq.(11) to Eq.(13).

$$
\begin{gathered}
D_{x}=\frac{\lambda \Delta y \Delta \mathrm{z}}{\Delta \mathrm{x}}, D_{y}=\frac{\lambda \Delta \mathrm{x} \Delta \mathrm{z}}{\Delta \mathrm{y}} \\
F_{x}=\rho c_{\mathrm{p}} u \Delta y \Delta z \\
F_{y}=\rho c_{\mathrm{p}} v \Delta \mathrm{x} \Delta z \\
D_{z}=\frac{\lambda \Delta \mathrm{x} \Delta \mathrm{y}}{\Delta \mathrm{z}}
\end{gathered}
$$


The system of equations formed by constructing equations resembling Eq.(2) for all the cells is then solved using a successively over relaxed Gauss-Seidel method.

\section{HYBRID FLUID DOMAIN THERMAL MODEL}

The current work focuses on eliminating the numerical discretization along the gap height, essentially transforming this $3 \mathrm{D}$ system to a $2 \mathrm{D}$ system. However, the error associated with the reduction of the grid size is unacceptable. In the proposed fast thermal solver, a hybrid scheme is used to capture the temperature distribution in the direction of film thickness analytically and in the rest two directions, numerically.

In order to capture the conduction on the solid boundaries considering the temperature distribution along the gap height, it is assumed that the temperature along the film thickness at a given $\mathrm{x}$ and $\mathrm{y}$ location varies quadratically. The rationale for this assumption is that the only mode of temperature transport along the film thickness is diffusion due to the absence of any kind of velocity along the same. This is also confirmed from Eq.(7) and Eq.(8), wherein the coefficients for the top and bottom neighbors (along the gap height) comprise only of diffusive factors.

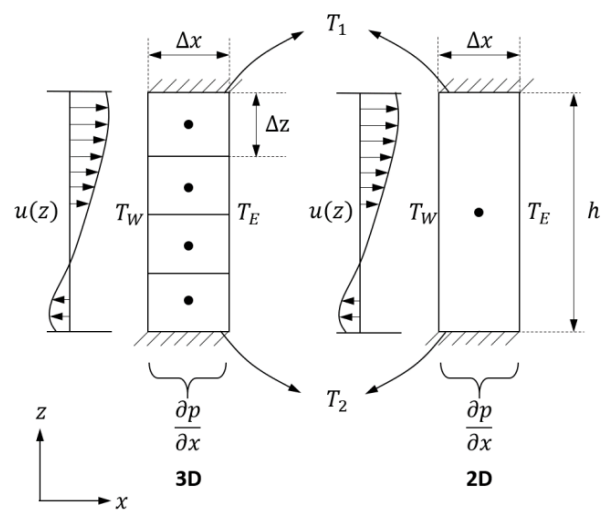

Figure 4 Simplified example

A simplified version of this idea is demonstrated by the example shown in Figure 4. The left part shows an analog of the 3D case discussed above and the right part shows a $2 \mathrm{D}$ analog to the proposed model. The 3D analog consists of finite volume cells along the z-axis which represents the film thickness. The dimension into the plane is taken to be infinite for the ease of demonstration. Each cell has dimensions $\Delta z$ along the $\mathrm{z}$-axis and $\Delta x$ along the $\mathrm{x}$-axis for the 3D analog and $h$ along the z-axis and $\Delta x$ along the $\mathrm{x}$-axis for the $2 \mathrm{D}$ analog ( $h$ represents the film thickness). Both the analogs have their top most and bottom most surfaces at fixed temperatures of $\mathrm{T}_{1}$ and $\mathrm{T}_{2}$ as shown. Both the examples have the same pressure gradient of $\frac{\partial \mathrm{p}}{\partial x}$ along the $\mathrm{x}$-axis. The velocity profile along the $\mathrm{z}$-axis, evaluated as a function of the pressure gradient and the velocities of the top and bottom most surface, are the same for both the analogs as shown. The temperatures on the right and left identical cells for both the analogs are represented by $\mathrm{T}_{\mathrm{W}}$ and $\mathrm{T}_{\mathrm{E}}$ respectively.

Equations similar to Eq.(2) to Eq. (13) are constructed for the 3D analog case and are shown from Eq.(14) to Eq. (21).

$$
\begin{aligned}
& D_{x 3 D}=\frac{\lambda \Delta z}{\Delta x} \\
& D_{z 3 D}=\frac{\lambda \Delta x}{\Delta z} \\
& F_{x 3 D}=\rho c_{p} u \Delta z \\
& a_{E 3 D}=D_{x 3 D} \\
& \cdot \max \left(0,\left(1-\frac{0.1\left|F_{x 3 D}\right|}{D_{x 3 D}}\right)^{5}\right) \\
& +\max \left(0,-F_{x 3 D}\right) \\
& \begin{array}{c}
a_{W 3 D}=D_{x 3 D} \\
\cdot \max \left(0,\left(1-\frac{0.1\left|F_{x 3 D}\right|}{D_{x 3 D}}\right)^{5}\right)
\end{array} \\
& +\max \left(0, F_{x 3 D}\right) \\
& a_{T 3 D}=D_{z 3 D} \\
& a_{B 3 D}=D_{z 3 D} \\
& b_{3 D}=\mu \Phi_{D} \Delta x \Delta \mathrm{z}
\end{aligned}
$$


A similar analysis is performed for the $2 \mathrm{D}$ analog and are shown from Eq.(27) to Eq. (32). The first obvious observation is that none of these equations have $\Delta z$ term in them. This is attributed to the elimination of the discretization along the film thickness. It can also be observed that the diffusion coefficient along the z-axis shown in Eq.(28), has a factor of 6 multiplied to it when compared to Eq.(15). This can be explained using Figure 5 and Figure 6. Figure 5 represents a more traditional linear slope approach wherein the value of the temperature of a cell is taken to be at the center and the slope at the top and bottom wall are simply taken as a linear variation from the wall temperature to the cell center temperature. Figure 6 represents a quadratic temperature profile where the temperature of a given cell, $T_{P}$, is taken to be the average of the quadratic temperature along the cell. The temperature profile is highlighted in blue. The temperature, as stated, is assumed to have a quadratic profile along the film thickness. This is represented by Eq.(22). The unknowns $A, B$ and $C$ are determined by the conditions shown in Eq.(23), Eq.(24) and Eq.(25). The resulting temperature profile is shown in Eq.(26). The factor 6 comes from the slope of this quadratic temperature profile at the top most and bottom most surface. The final change is that the coefficients for the top and bottom neighbors are eliminated. This is accounted for by having an extra term in the source term shown in Eq.(32). This extra term accounts for the diffusion along the $\mathrm{z}$-axis.

$$
\begin{gathered}
T(z)=A z^{2}+B z+C \\
T(0)=T_{2} \\
T(h)=T_{1} \\
\frac{1}{h} \int_{0}^{h} T(z) d z=T_{P} \\
T(z) \quad\left[\frac{-3\left(2 T_{P}-T_{1}-T_{2}\right)}{h^{2}}\right] z^{2} \\
+\left[\frac{2\left(3 T_{P}-T_{1}-2 T_{2}\right)}{h}\right] z+T_{2}
\end{gathered}
$$

$$
\begin{aligned}
& D_{x 2 D}=\frac{\lambda h}{\Delta x} \\
& D_{z 2 D}=\frac{6 \lambda \Delta x}{h} \\
& F_{x 2 D}=\rho c_{p} u_{a v g} h \\
& a_{E 2 D}=D_{x 2 D} \\
& \cdot \max \left(0,\left(1-\frac{0.1\left|F_{x 2 D}\right|}{D_{x 2 D}}\right)^{5}\right) \\
& +\max \left(0,-F_{x 2 D}\right) \\
& a_{W 2 D}=D_{x 2 D} \\
& \cdot \max \left(0,\left(1-\frac{0.1\left|F_{x 2 D}\right|}{D_{x 2 D}}\right)^{5}\right) \\
& +\max \left(0, F_{x 2 D}\right) \\
& b_{2 D}=\mu \Phi_{D} \Delta x h+D_{z 2 D} \cdot\left(T_{1}+T_{2}\right)
\end{aligned}
$$

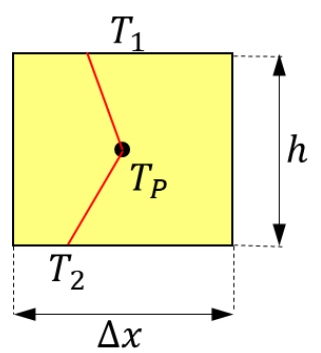

Figure 5 Traditional linear slope

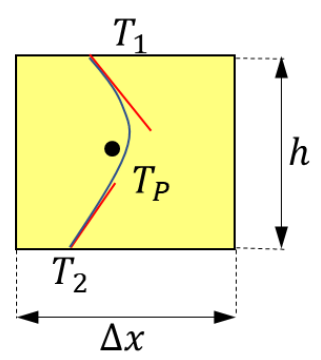

Figure 6 Quadratic slope 
The 3D analog model solved for the temperature profile along the $\mathrm{z}$-axis and the $2 \mathrm{D}$ analog model solves for the average temperature. In order to show a comparison of the results obtained using the two approaches, the difference of the average temperature solved using the $2 \mathrm{D}$ analog model and the average of the temperature profile obtained using the 3D analog model, for a range of gap heights and pressure gradients is shown in Figure 7. This case pertains to no velocity on both the top and bottom surfaces, the right and left neighbor temperatures as $50^{\circ} \mathrm{C}$ and $40^{\circ} \mathrm{C}$, and the top and bottom surfaces are both at $50^{\circ} \mathrm{C}$. It is observed that in general, both the analogs give similar average temperatures owing to the difference being around a null value everywhere. However, it is observed that for higher values of pressure gradients and gap heights, the 2D analog slightly overpredicts the temperature in comparison to the $3 \mathrm{D}$ analog. This is attributed to the fact that the velocity used for the determination of the convection coefficient in Eq.(29) is an average velocity. This underestimates the thermal energy convected out of the cell when the velocities are higher. It is noted here that similar trends were observed for different boundary temperatures and velocities.

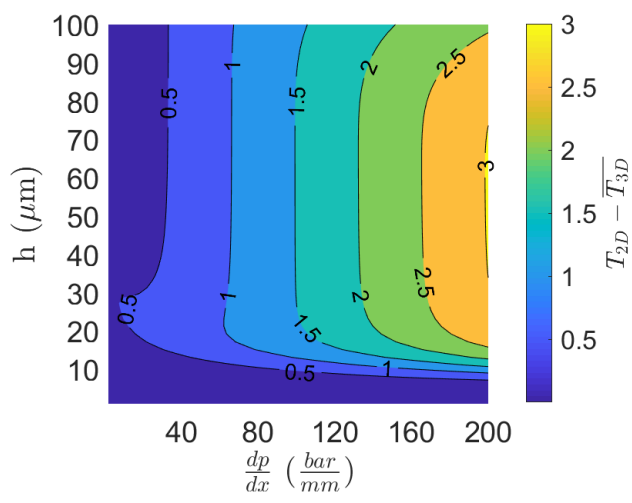

Figure 7 Difference of 2D and mean 3D temperatures

In order to account for the overprediction of temperatures at high velocities, the convection coefficient shown in Eq.(29), was multiplied with a correction factor $f_{c}$. To estimate the value of $f_{c}$, an optimization study was performed such that the difference of the temperatures as shown in Figure 7 is close to zero. A plot of this coefficient $f_{c}$, as a function of the pressure gradient and gap height, for the same conditions as that of Figure 7, is shown in Figure 8.

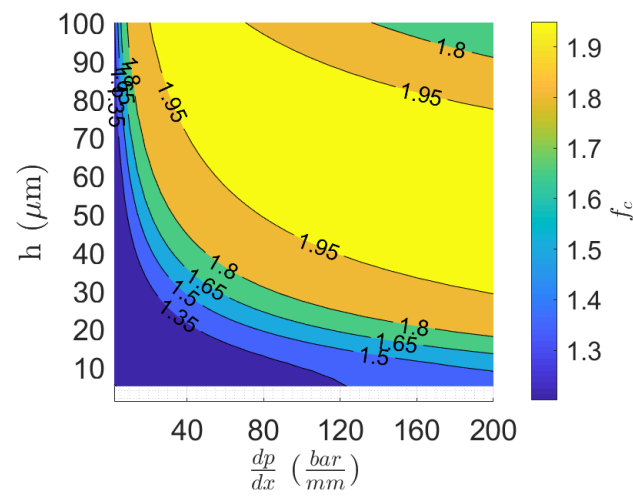

Figure 8 Convection coefficient correction factor

It is observed that the value of $f_{c}$ for very small film thicknesses is not shown. This is attributed to the fact that at very low film thicknesses, where the velocity is low, the optimization fails to find a meaningful coefficient. For the rest of the space, a trend is observed with the correction coefficient, $f_{c}$. It is observed that as the amount of convective transport increases with either the pressure gradient or the film thickness, this coefficient rises from a value close to 1 , to a value close to 2 . At very high-pressure gradients and film thicknesses, the coefficient is seen to again drop but it is noted that pressure gradients of 200 $\mathrm{bar} / \mathrm{mm}$ over gap heights of 100 microns is not physical in the lubrication regimes. Similar trends were obtained for different boundary temperatures and velocities as well.

A non-dimensional number that accounts for the combined effect of the film thickness and the pressure gradient is the Peclet number, shown in Eq.(33). Peclet number, $P e$, is the ratio of the convective flux to the diffusive flux. It was observed that the variation of the correction factor, $f_{c}$, with the film thickness and the pressure gradient can be captured using the Peclet number, $\mathrm{Pe}$.

$$
P e=\frac{F_{x 2 D}}{D_{x 2 D}}
$$


Figure 8 can also be interpreted through $P e$. A line of constant pressure gradient and a line of constant film thickness is taken from Figure 8 and the variation of $f_{c}$ is plotted along them as a function of $P e$ as shown in Figure 9. It is observed that both the lines represent the same values of $f_{c}$ as a function of $P e$ along them. This confirms the fact that the correction factor depends on $P e$. In order to have a generic expression of $f_{c}$ as a function of $P e$, the value of $f_{c}$ is said to be 1 for very small Peclet numbers and 2 for very high Peclet numbers. The switch from 1 to 2 occurs smoothly across a correction value of 1.5 which was observed to occur at a Peclet number of $10^{5}$. This is functionally obtained using a hyperbolic tangent function as shown in Eq.(34), where $P e_{\text {lim }}$ is the limiting Peclet number of $10^{5}$ where $f_{c}$ attains a value of 1.5.

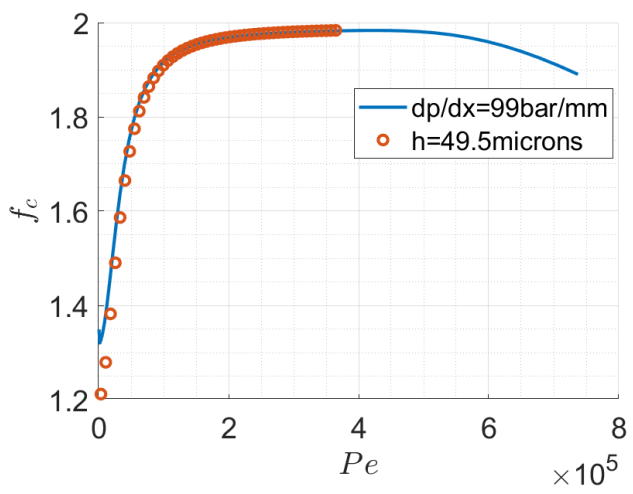

Figure 9 Correction factor as a function of $\mathrm{Pe}$

$$
f_{c}=1.5+\frac{\tanh \left(\frac{P e-P e_{\text {lim }}}{P e_{\text {lim }}}\right)}{2}
$$

A comparison of the optimized correction factor and the correction factor derived in Eq.(34) is shown in Figure 10. It is observed that the derived $f_{c}$ is slightly different from the optimized $f_{c}$. This is attributed to the fact that the optimized $f_{c}$ was obtained using an optimization algorithm and the results need not be exactly perfect. The optimization algorithm tends to find the best possible coefficient to minimize the error between the $2 \mathrm{D}$ and $3 \mathrm{D}$ temperatures. It was observed that the optimized coefficient, although having a similar trend and bounding values, had slightly different slopes for different operating conditions. In order to make the derived coefficient, $f_{c}$, independent of operating parameters other than $P e$, the hyperbolic tangent function was chosen such that it follows the same trend and bounding values as the optimized coefficient but differs slightly in terms of the slope of the function. Figure 11 and Figure 12 show the difference between the 2D temperatures and mean 3D temperatures, similar to Figure 7, using the optimized and derived convection correction factors. It is observed that although the differences in Figure 12 are not as low as those in Figure 11, the differences are still very small. It is observed that the difference is relatively higher in regions of very high pressure gradients, it is noted that such high pressure gradients over a single cell is usually not seen.

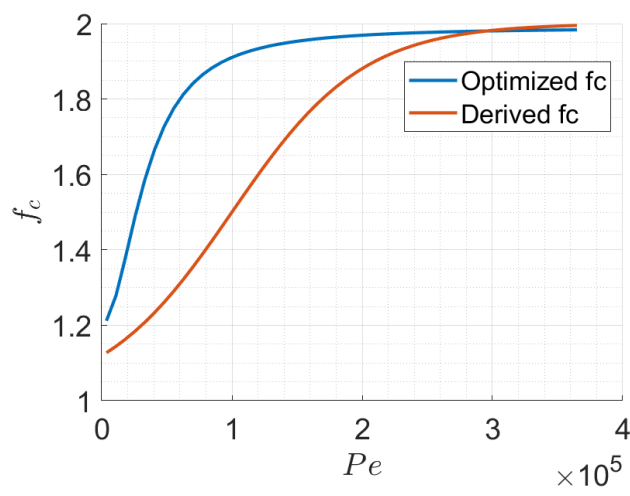

Figure 10 Derived correction factor 


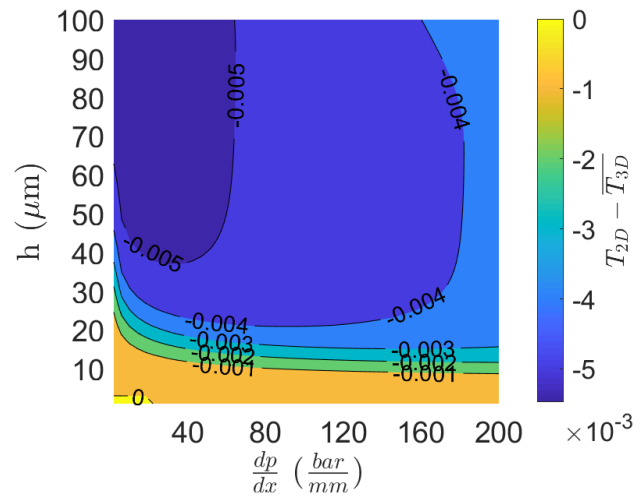

Figure 11 Difference of 2D and mean 3D temperatures using optimized $\mathrm{fc}$

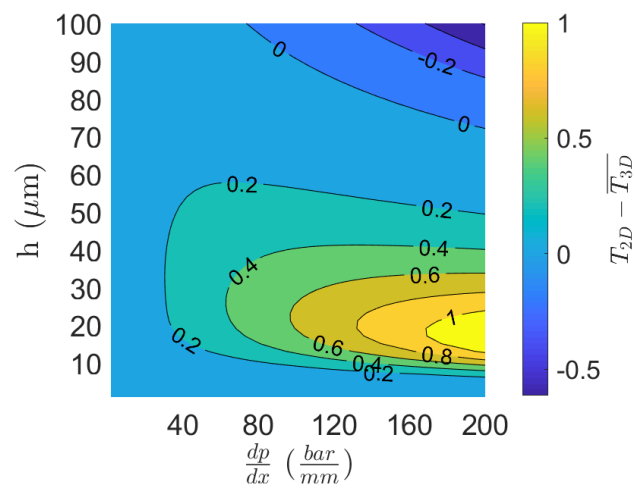

Figure 12 Difference of 2D and mean 3D temperatures using derived $\mathrm{fc}$

This concept is applied to the energy equation solver in the interface between the piston and cylinder. The grid used for the new 2D solver is shown in Figure 13. It is to be noted that this grid is different from the one shown in Figure 2 in the sense that there is no discretization along the film thickness. The modified finite volume cell for the unwrapped 2D grid is shown in Figure 14. It is observed that this cell has a dimension of $h$, the film thickness, along the z-axis. The top and bottom neighbors are absent due to the reduction in dimension. The top wall $t$ and bottom wall $b$ are at fixed temperatures corresponding to the bounding solids, piston and bushing running surfaces in this case. The parameters along the other two dimensions: $x$ and $y$ remain the same.
The modified coefficients for this cell are shown from Eq.(35) to Eq.(42). $f_{c x}$ and $f_{c y}$ represent the discussed correction factors in the $\mathrm{x}$ and $\mathrm{y}$ directions respectively.

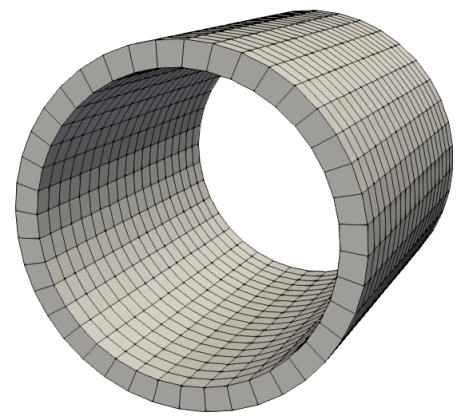

Figure 13 2D FVM grid

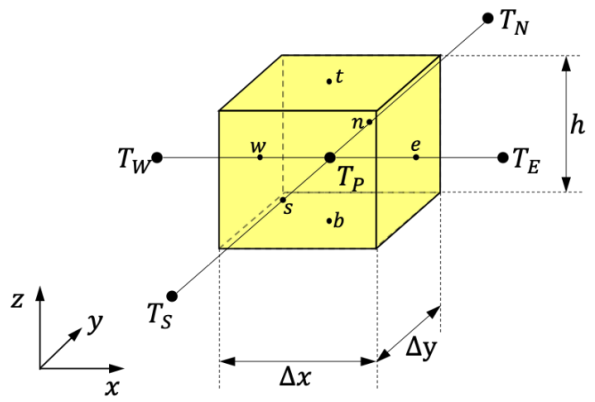

Figure 14 FVM cell from 2D grid

$$
\begin{gathered}
D_{x}=\frac{\lambda \Delta y}{\Delta \mathrm{x}} h, D_{y}=\frac{\lambda \Delta \mathrm{x}}{\Delta \mathrm{y}} h \\
F_{x}=f_{c x} \cdot \rho c_{\mathrm{p}} u \Delta y h, \\
F_{y}=f_{c y} \cdot \rho c_{\mathrm{p}} v \Delta \mathrm{x} h \\
D_{z}=\frac{6 \lambda \Delta \mathrm{x} \Delta \mathrm{y}}{h}
\end{gathered}
$$




$$
\begin{aligned}
& a_{E}=D_{x} \cdot \max \left(0,\left(1-\frac{0.1\left|F_{x}\right|}{D_{x}}\right)^{5}\right) \\
& +\max \left(0,-F_{x}\right) \\
& a_{W}=D_{x} \cdot \max (0,(1 \\
& \left.\left.-\frac{0.1\left|F_{x}\right|}{D_{x}}\right)^{5}\right) \\
& +\max \left(0, F_{x}\right) \\
& a_{N}=D_{y} \cdot \max (0,(1 \\
& \left.\left.-\frac{0.1\left|F_{y}\right|}{D_{y}}\right)^{5}\right) \\
& +\max \left(0,-F_{y}\right) \\
& a_{S}=D_{y} \cdot \max \left(0,\left(1-\frac{0.1\left|F_{y}\right|}{D_{y}}\right)^{5}\right) \\
& +\max \left(0, F_{y}\right) \\
& b=\mu \Phi_{D} \Delta x \Delta \mathrm{y} \Delta \mathrm{z}+\mathrm{D}_{\mathrm{z}} \cdot\left(T_{t}+T_{b}\right)
\end{aligned}
$$

\section{RESULTS}

In order to show the potential of the proposed formulation, a corner operating condition of 3600 rpm, 450 bar and $100 \%$ displacement was chosen for a reference $75 \mathrm{cc}$ axial piston unit. The higher speeds lead to higher energy dissipation and the high pressure increases the convection of energy in the fluid. The high displacement ensures large fluctuations in the fluid temperatures. For the purposes of comparison, a fluid grid with 80 cells along the circumferential direction, 40 cells along the axial direction and 30 cells along the film thickness is chosen.

Figure 15 shows the average temperature along the film thickness, over an unwrapped film at a shaft angle of $90^{\circ}$ obtained using the 3D energy equation. This profile will be used as a reference to compare the temperatures obtained using the 2D model.

In order to demonstrate a step by step evolution of the 2D model, the temperature field obtained using the 3D model, with 2 cells along the film thickness is shown in Figure 16. It is observed that this evaluation does not yield to a meaningful temperature field. The temperature in the lower portion of the film is underpredicted to a degree that does no longer represents the physics of the film properly. The temperature field obtained using the 2D energy equation, without the use of a quadratic slope, as described in Eq.(28), and without any convection correction, as described in Eq.(34), is shown in Figure 17. It is observed by comparing Figure 15 and Figure 17, that the 2D model overpredicts the average temperature in the lower portion of the film and underpredicts the average temperature in the upper portion of the film. This mismatch is explained by referring to Figure 5 and Figure 6. The temperature field in Figure 17 corresponds to the linear slope. This formulation does not actually account for the physically correct diffusion through the top and bottom surfaces. 


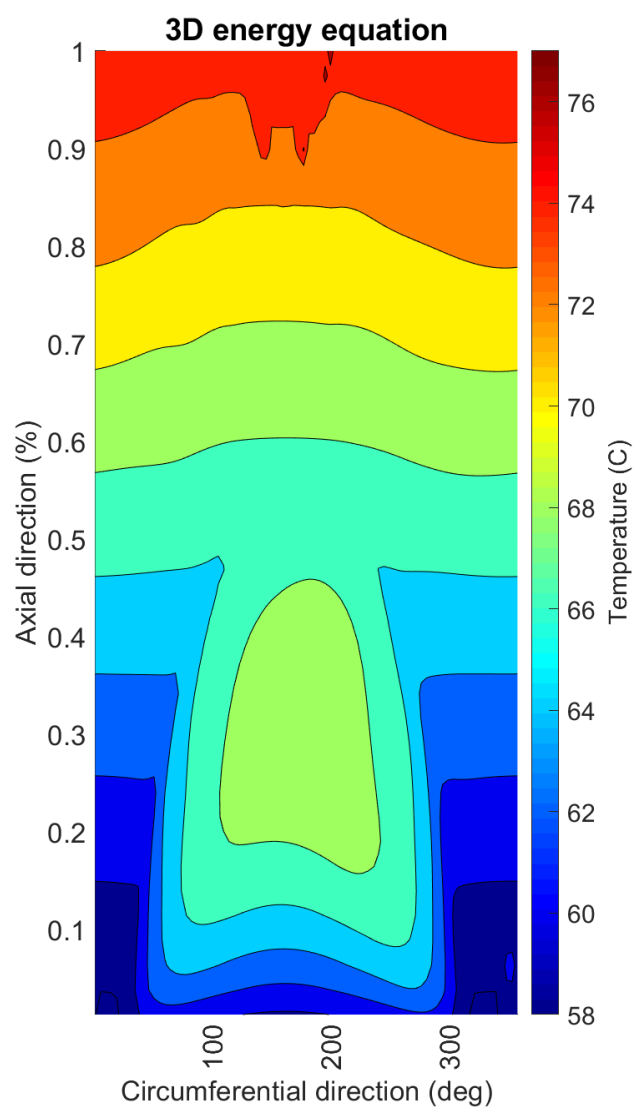

Figure 15 Unwrapped film temperature from 3D energy equation

The temperature profile over the unwrapped film thickness, obtained using the 2D energy equation with the modified quadratic slope and no convection correction is shown in Figure 18. A comparison of this profile with Figure 15 shows that the underprediction in the upper portion of the film is not present. This is attributed to the modified slope which ensures that the right amount of energy is diffused through the bounding surfaces of the fluid film. It is observed that the overprediction in the lower portion of the film has reduced compared to Figure 17, although the temperature is still overpredicted. This is attributed to the film thickness and average axial velocity as shown in Figure 19 and Figure 20 respectively. The film thickness in the region of overprediction in the lower portion of the fluid film is relatively high. Combined with the fact that the axial velocity is high in this region, this leads to a higher Peclet number as discussed in the previous section. This leads to the underprediction of the convective flux of energy in this region leading to an overprediction of temperature. The correction factor, as shown in Eq.(34), is required to account for this phenomenon. It is also noted that the gap height is higher in other regions of the film, as the upper left and right sections. Although, the axial velocity in these regions is not as high as it is in the central portion and hence leading to a lower value of Peclet number resulting in reasonable prediction of temperature.

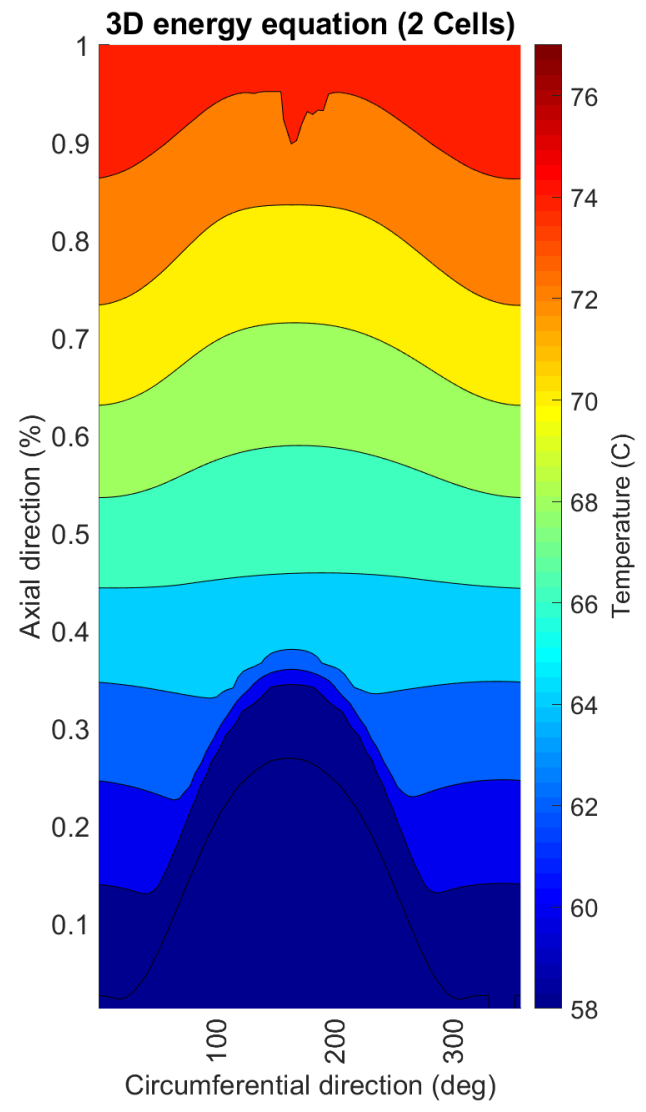

Figure 16 Film temperature from 3D energy equation using 2 cells 


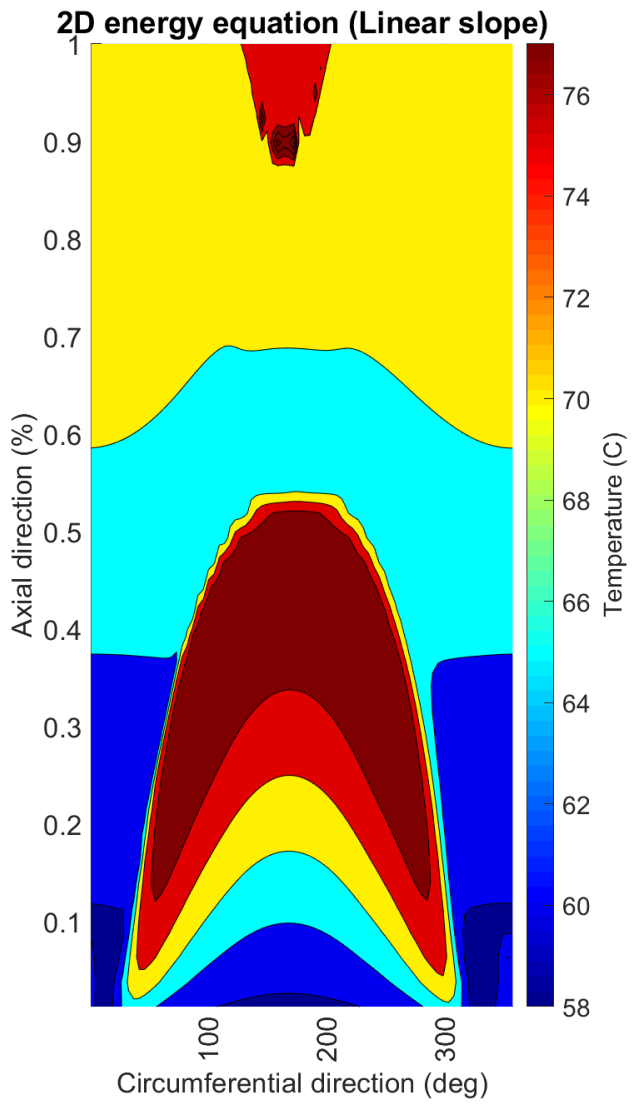

Figure 17 Film temperature from 2D energy equation with linear slope at the walls

The temperature profile obtained using the final 2D model accounting for the quadratic slope and the convective correction is shown in Figure 21. It is observed that the temperature profile is much closer to Figure 15 than the ones shown in Figure 17 and Figure 18. Although, there is a slight difference in the central portion of the film, the overall temperature in the film seems closely similar to Figure 15. The proposed model resulted in an average temperature difference of $2 \%$ throughout the film in comparison to the $3 \mathrm{D}$ model. The important factor here is that the time required to evaluate the $3 \mathrm{D}$ model till convergence was around 7.2 hours, whereas the $2 \mathrm{D}$ evaluation required around 4.6 hours. The $2 \mathrm{D}$ model is approximately $36 \%$ faster than the $3 \mathrm{D}$ model.

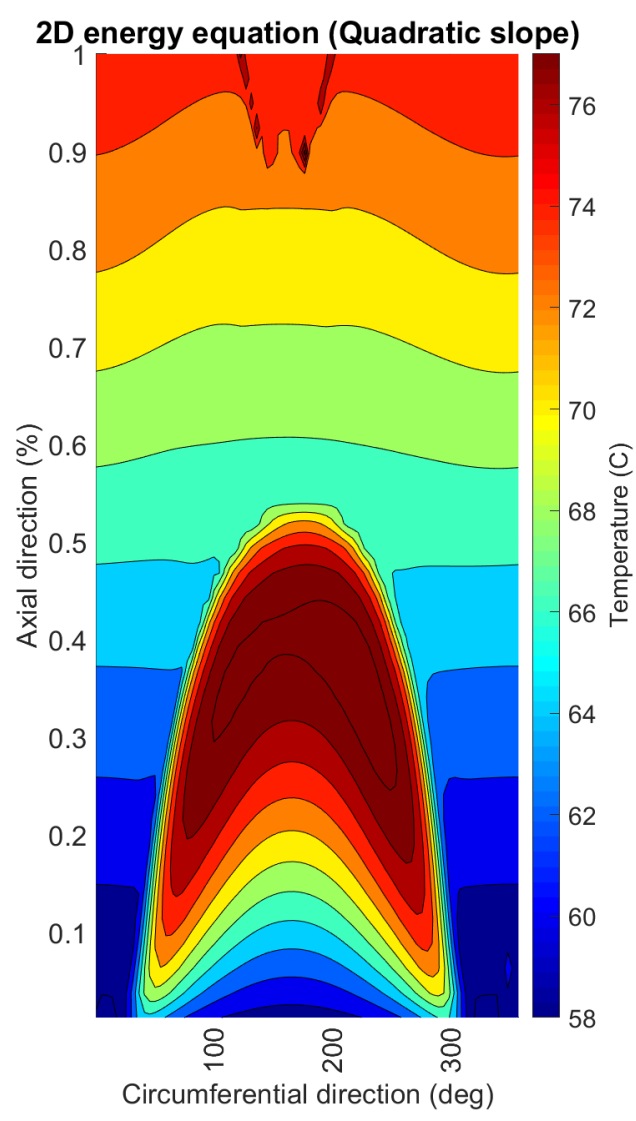

Figure 18 Film temperature from 2D energy equation with quadratic slope at the walls 


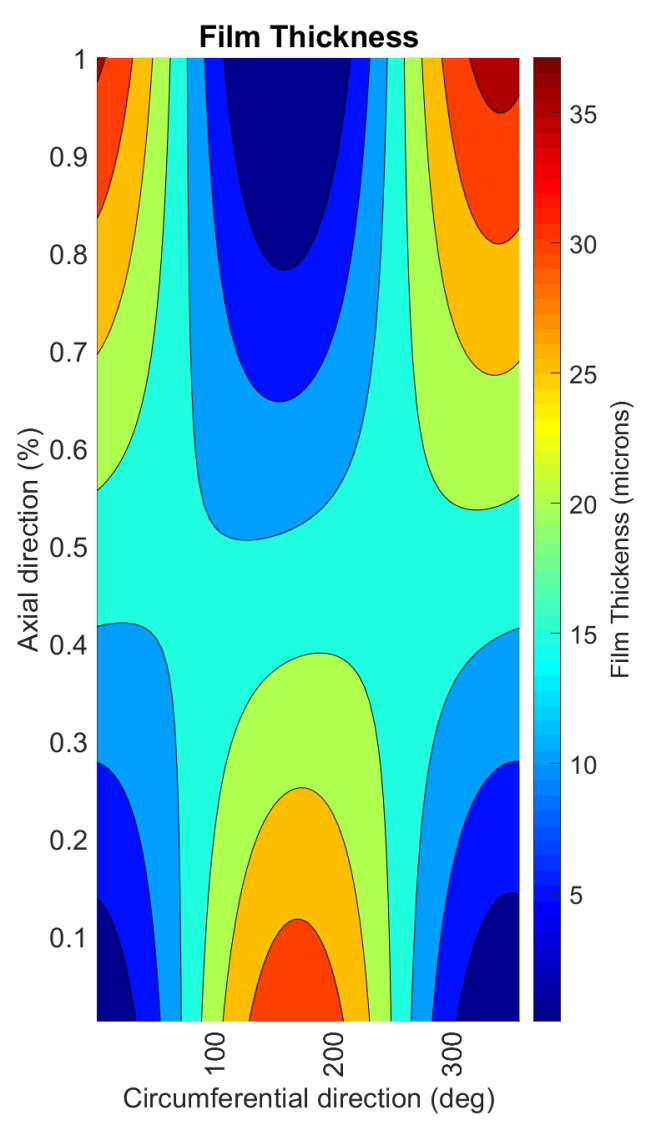

Figure 19 Film thickness over the unwrapped fluid film

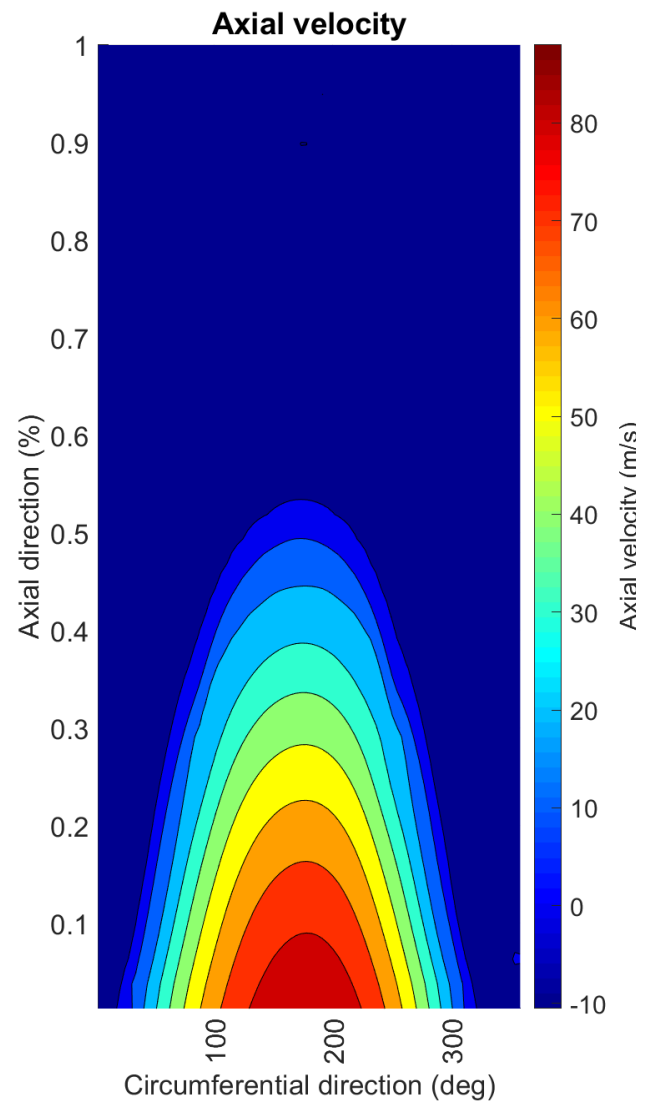

Figure 20 Average axial velocity over the unwrapped fluid film 


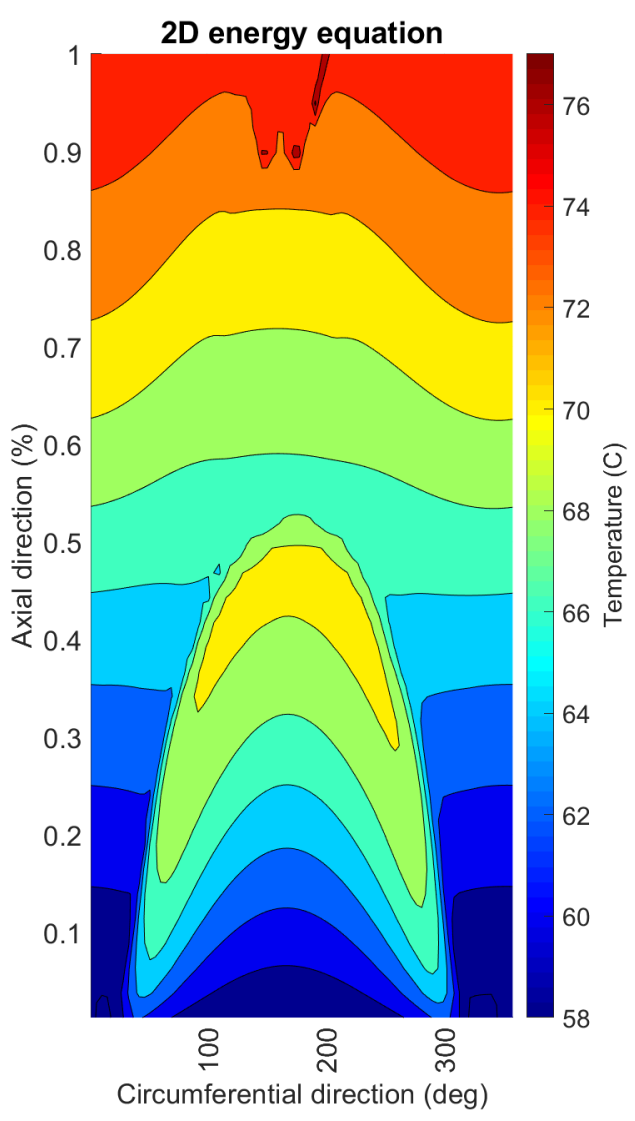

Figure 21 Unwrapped film temperature from 2D energy equation

\section{CONCLUSION}

A 2D energy equation model was proposed with the assumption of a parabolic temperature distribution along the gap height. New finite volume coefficients were derived for the proposed model along with a derived correction factor for the convective terms. The average difference between the 3D model and the proposed model was not observed to be more than $2 \%$, but a speedup of $36 \%$ was observed in terms of simulation times. It is to be noted that although the model was demonstrated for the piston/cylinder interface of an axial piston machine, it can be applied to any lubricating interface simulation tool to reduce the simulation time taken to solve the energy equation.

\section{NOMENCLATURE}

$\begin{array}{ccc}\rho & \text { Fluid density } & \mathrm{Kg} / \mathrm{m}^{3} \\ c_{p} & \text { Specific heat } & \mathrm{J} / \mathrm{kg} \mathrm{K} \\ u & \text { x-velocity } & \mathrm{m} / \mathrm{s} \\ v & \text { y-velocity } & \mathrm{m} / \mathrm{s} \\ T & \text { Temperature } & { }^{\circ} \mathrm{C} \\ \lambda & \text { Fluid conductivity } & \mathrm{W} / \mathrm{m} \mathrm{K} \\ \mu & \text { Fluid viscosity } & \mathrm{Pa} \mathrm{s} \\ \phi & \text { Viscous dissipation } & \mathrm{W} / \mathrm{kg} \\ P e & \text { Peclet number } & -\end{array}$

\section{REFERENCES}

[1] H. G. Elrod, D. E. Brewe, L. Rt, and H. G. Elrod, "Technical Memorandum 88845 Technical Report 86-C-33 Thermohydrodynamic Analysis for Laminar Lubricating Films," 1986.

[2] V. der Kolk, "Beitrag zur Bestimmung der Tragfähigkeit des stark verkanteten Gleitlagers Kolben - Zylinder an Axialkolbenpumpen der Schrägscheibenbauart," University of Karlsruhe, 1972.

[3] A. Yamaguchi, "Motion of Pistons in PistonType Hydraulic Machines," Bull. JSME, vol. 19, no. 130, pp. 402-419, 1976.

[4] M. Ivantysynova (at that time Berge), “An investigation of viscous flow in lubricating gaps. (In Slovak)," SVST Bratislava, Czechoslovakia, 1983.

[5] L. Olems, "Ein Beitrag zur Bestimmung des Temperaturverhaltens der KolbenZylinderbaugruppe von Axialkolbenmaschinen der Schrägscheibenbau-weise," VDI Fortschrittsberichte, vol. Reihe 1, no. 348, 2001.

[6] U. Wieczorek and M. Ivantysynova, "Computer aided optimization of bearing and sealing gaps in hydrostatic machinesthe simulation tool caspar," Int. J. Fluid Power, vol. 3, no. 1, pp. 7-20, 2002.

[7] M. IVANTYSYNOVA and C. HUANG, "Investigation of the Gap Flow in Displacement Machines Considering Elastohydrodynamic Effect," Proc. JFPS Int. Symp. Fluid Power, vol. 2002, no. 5-1, pp. 219-229, 2002.

[8] M. Pelosi and M. Ivantysynova, "A novel fluid-structure interaction model for lubricating gaps of piston machines," WIT Trans. Built Environ., vol. 105, pp. 13-24, 2009. 
[9] M. Pelosi and M. Ivantysynova, "A Geometric Multigrid Solver for the PistonCylinder Interface of Axial Piston Machines," Tribol. Trans., vol. 55, no. 2, pp. 163-174, 2012.

[10] L. Shang and M. Ivantysynova, "Port and case flow temperature prediction for axial piston machines," Int. J. Fluid Power, vol. 16, no. 1, pp. 35-51, 2015.

[11] L. Shang and M. Ivantysynova, "Advanced heat transfer model for piston/cylinder interface," 11th IFK Int. Fluid Power Conf., vol. 1, no. 1972, pp. 587-595, 2018.

[12] H. McCallion, F. Yousif, and T. Lloyd, "The analysis of thermal effects in a full journal bearing," J. Tribol., vol. 92, no. 4, pp. 578587, 1970 .

[13] J. Ferron, J. Frene, and R. Boncompain, "A study of the thermohydrodynamic performance of a plain journal bearing comparison between theory and experiments," J. Tribol., vol. 105, no. 3, pp. 422-428, 1983.

[14] Y. Song, J. Ma, and S. Zeng, "A numerical study on influence of temperature on lubricant film characteristics of the piston/cylinder interface in axial piston pumps," Energies, vol. 11, no. 7, 2018.

[15] M. Pelosi, "An investigation of the fluidstructure interaction of piston/cylinder interface," 2012. 